\title{
Extending WS-Agreement to Support Automated Conformity Check on Transport and Logistics Service Agreements ${ }^{\star}$
}

\author{
Antonio Manuel Gutiérrez ${ }^{1}$, Clarissa Cassales Marquezan ${ }^{2}$, Manuel Resinas $^{1}$, \\ Andreas Metzger ${ }^{2}$, Antonio Ruiz-Cortés ${ }^{1}$, and Klaus Pohl ${ }^{2}$ \\ 1 School of Computer Engineering \\ University of Seville \\ \{amgutierrez, resinas, aruiz\} @us.es \\ 2 Paluno - University of Duisburg-Essen, Essen, Germany \\ $\{$ clarissa.marquezan, andreas.metzger, klaus.pohl\}@paluno.uni-due.de
}

\begin{abstract}
Checking whether the agreed service quality attributes are fulfilled or maintained during the service life-cycle is a very important task for SLA (Service Level Agreement) enforcement. In this paper, we leverage conformance checking techniques developed for computational services to automate the conformity checking of transport \& logistics services. Our solution extends the WS-Agreement metamodel to support the definition of frame and specific SLAs. With this extension, we define a new validation operation for the conformity check of transport \& logistics SLAs based on CSPs solvers. The key contribution of our work is that, as far as we know, it is the first definition of an automated conformity check solution for long term agreements in the transport \& logistics domain. Nonetheless, other domains in which similar SLAs are defined can also benefit from our solution.
\end{abstract}

\section{Introduction}

Service Level Agreements (SLAs) are essential in service provision because they define the quality attributes of services meeting consumers and providers preferences. These quality attributes or SLOs (Service Level Objectives) describe in a measurable way how the service should behave during its life-cycle or what the basic requirements for its execution are. Checking whether the agreed SLOs are fulfilled or maintained is a very important task for SLA enforcement.

In transport \& logistics services, one type of SLA commonly used by large companies establishes an interval of time in which multiple executions of the same service will be requested by the Logistic Service Client (LSC) and executed by the Logistic Service Provider (LSP). This type of SLA comprises one

\footnotetext{
* This work was partially supported by the European Union's Seventh Framework Programme (FP7/2007-2013), the European Commission (FEDER), the Spanish and the Andalusian R\&D\&I programmes (grant agreements 215483 (S-Cube), 285598 (FInest) and 604123 (FIspace), TIN2012-32273 (TAPAS), TIC-5906 (THEOS)).
} 
frame agreement document and multiple "child" agreements, i.e., for each LSC request a new agreement will be created eventually containing the same SLOs from its parent frame agreement. Currently, a manual process is used to check whether the SLOs of "child" agreements (called specific agreements) conform with the SLOs of the frame agreement. Numbers from a large company from the transport \& logistics domain [8] show that in a random month approximately 100,000 transportations happen. We can say that each transportation might be associated with a specific agreement. This means that the number of specific agreements to be checked by a large company could reach up to 100,000 documents per month. Thus, our goal is to automate this checking by proposing a conformity check solution for SLAs in the transport \& logistics domain.

Conformity checks in SLAs of computational services, like Cloud services and SBAs (Service Based Applications), have been extensively proposed. Two main groups of proposals can be found: One group aims at checking whether the execution of a service conforms with the SLOs on the SLA document [2]. The other group focuses on supporting consistent SLA definition, avoiding errors or inconsistent terms between documents 104. Nevertheless, when it comes to SLAs of real physical services like shipment of goods in the transport \& logistics domain, the current conformity check solutions lack a proper handling of this type of SLAs. The main reason for this are the differences between SLAs in the transport \& logistics and computational SLAs: the existence of two levels of agreements and the need to aggregate information between these levels to perform conformity check. Current works fall short on addressing these differences.

In this paper, we propose a solution able to handle the aforementioned differences and provide the automated conformity check of SLAs in transport \& logistics domain. Our proposal is based on WS-Agreement which is a standard that is widely used and that has been successfully applied in the computational domain [10]. However, it currently lacks the necessary formalization to represent the relationship among the two levels of SLAs in transport \& logistics, i.e., frame and specific agreements. Therefore, we extend the WS-Agreement metamodel to fill this gap. With these extensions, we can define a new validation operation for the conformity check of transport \& logistics SLAs. This new operation extends previous work 109] which were targeted only at computational SLAs. In that work, SLOs are mapped to Constraints Satisfaction Problems (CSPs) and the conformity check is achieved using CSP solvers. We validate our proposal with the implementation of a too 1 . The key contribution of our work is that, as far as we know, it is the first definition in the literature of an automated conformity check solution for SLAs in the transport \& logistics domain. Furthermore, since our conformity checking is domain independent, other domains in which similar long-term agreements are defined can also benefit from our solution.

The remaining of this paper is organized as follows. In Section 2 we describe the related work. Section 3 presents the WS-Agreement extension proposed in this paper. Section 4 presents the new operations defined in order to perform the conformity check. In Section 5 we discuss the conclusions and future work.

${ }^{1}$ http://www.isa.us.es/tlcc 


\section{Related Work}

In the past years, there has been an increasing amount of research efforts trying to bring into the transport \& logistics domain technical solutions based on Service Oriented Computing 2 . Nevertheless, when it comes to SLAs and conformity check in this domain, there is a limited amount of work. The solution of Augenstein et al. 1 introduce a platform based on a service-oriented approach for managing contracts on $4 \mathrm{PL}$ busines 3 . The proposed solution itself is mainly focused on coordinating the business process conducted among these different partners. Another example is the work introduced by Bing and Zhongying [3]. They use mathematical terms to define the parameters of a contract in transport \& logistics collaborative business process. Mai and Teo [7] also followed a mathematical approach to define and analyze contracts in the collaborative business process in transport \& logistics. Nevertheless, none of the aforementioned solutions focus on the conformity check of the agreements among the partners.

As previously discussed, the major differences between services in transport $\&$ logistics to computational services are the existence of two levels of agreements and the need to aggregate and compare information between these levels to perform conformity check. In this section, we show how current works on conformity check fall short on addressing these differences. The work proposed by Leitner et al. 6] aims at predicting SLA violations in business process. The authors consider two types of SLOs: instance-level, associated with each instance of a business process in isolation; and aggregated, representing the execution of several instances of the same type of business process. The violation prediction of aggregated SLOs is performed on values at the same SLA level, i.e., the same type of document describing the SLOs of a business process. In our case, we need to aggregate information at the same level, but in contrast, we need to compare this information to a different level of SLA document. This will be discussed in detail in the next section.

Bartoline et al. [2] proposed to monitor the QoS attributes of service choreographies to detect violations on the choreography SLA. The authors present a new approach to annotate the BPMN Choreography Diagram with functional and non-functional constraints that need to be fulfilled by a service entering the choreography. At design time, these constraints are analyzed and translated into monitoring rules to be used during runtime. Goel et al. [5] use temporal logics of safety (DSF - Deterministic Safety Formula) to formalize the SLOs and model checking to support the monitoring conformance of SLAs. Their solution is able to detect and present to the user the occurrence of violations of the specified SLA. The aforementioned approaches do not deal with the conformance checking between frame and specific agreements and they have limited support for expressing aggregate information in SLOs.

\footnotetext{
2 http://www.finest-ppp.eu/files/deliverables/d08/finest_d8_1_final.pdf

3 4PL business (4th Party Logistics) or 3PL (3rf Party Logisitcs) are types of business processes that result in a supply chain with collaborative tasks executed by different logistics partners.
} 


\section{Modelling Long Term Transport and Logistics Agreements}

The first step towards supporting automated checks is to model frame and specific agreements so that their constraints can be checked by a software component. A limitation of the AS-IS situation in transport \& logistics is that there is no explicit mechanism to represent the aggregation of specific agreements by the frame agreement. However, as introduced in Section 1, some types of SLO values defined in the specific agreements are actually constrained by the SLOs defined in the frame agreement and the SLOs of the specific agreements that have been already signed. This is the case of, e.g., an SLO "maximum containers" specified in a frame agreeement to limit the total amount of containers transported in a time period. This SLO affects to SLOs related to number of containers transported in every specific agreement under the frame agreement context.

We propose modelling the relationship between the SLOs of frame agreements and specific agreements by clearly defining the existence of what we called: atomic and aggregated SLOs. The SLO type defines which kind of constraints have to be applied during the conformity check and this allows to identify and create the explicit link between frame and specific agreement. All types of contracts have atomic attributes. Values are assigned to these attributes at the contracting phase of the service and do not change over time unless they are explicitly renegotiated by the parties. In contrast, aggregated attributes are only associated with frame agreements and their values depend on the values of atomic attributes from specific agreements associated with the frame agreement.

WS-Agreement specification provides an agreement document schema so it can be used to define frame and specific agreement. Specific agreements and atomic SLOs can be directly defined with this model. For instance, Figure 1 depicts an specific agreement modelled in WS-Agreement. However, there is no mechanism to model which specific agreements are associated with the frame agreement nor to define aggregated SLOs so frame agreements cannot be directly modelled with WS-Agreement. Therefore, in this proposal we extend WSAgreement to be able to model frame agreements.

First, we extend the WS-Agreement context with one additional section (cf. Figure1) to include all the specific agreements validated in the frame agreement

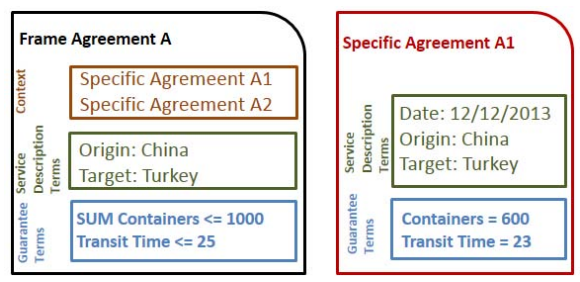

Fig. 1. WS-Agreement modelling of Frame Agreements and Specific Agreements 
context. Second, in order to define aggregated SLOs, accumulative operators are introduced. Thus, the example "maximum containers" SLO could be defined as:

\section{Guarantee Terms: Maximum Containers: Provider guarantees SUM (SpecificAgreement.Containers) < 1000}

where SpecificAgreement refers to the specific agreements whose conformity with this frame agreement has been evaluated and SUM is the summation operator. A set of aggregation operators such as COUNT, MAX and MIN could be used. These extensions enable the definition of the aggregation SLOs (Figure 1).

\section{Automated Validation of Specific Agreements}

The validation of a specific agreement in the context of a frame agreement involves checking their conformity. Automating conformity checking between frame agreement and specific agreement makes possible to detect errors in early stages. So, once a frame agreement has started its validity period, each time a new specific agreement is signed, the conformity between its SLA terms and frame agreement can be checked. This check operation is described in this section.

Two types of conformance issues may appear between specific and frame agreements. On one hand, specific agreements may include atomic SLOs that violate atomic SLOs defined in the frame agreement (e.g. if the frame agreement determines a transit time limit of 25 days, a transit time of 30 days in specific agreement would not be conform). On the other hand, specific agreements may also violate aggregated SLOs (e.g. if the frame agreement defines an aggregation SLO "maximum containers = 100", specific agreements with SLO "containers" are not conform to the frame agreement if its atomic SLO "containers" plus the atomic SLO "containers" from previous specific agreements is more than 100).

From this discussion, we conclude that valid values for any service property used in specific agreement SLOs have to be also valid for frame agreement SLOs (not the opposite). Consequently, the conformance between a specific and a frame agreement conformity can be informally defined as follows: "a specific agreement conforms to a frame agreement if the set of possible values for the service properties used in its SLOs is a subset of the possible values for the service properties used in the SLOs in the frame agreement".

CSP Mapping. Following this notion of conformance, we follow an approach similar to [10 to automate its checking. The procedure involves mapping both the frame agreement and the specific agreement into a Constraint Satisfaction Problem (CSP) and use a CSP solver to check the conformance between them. The difference being that, in our case, it is necessary to take the aggregated SLOs and previous specific agreements into account.

There are several reasons for choosing CSPs to automate this checking. First, an important part of the agreement (SDT and GT) are described as constraints 


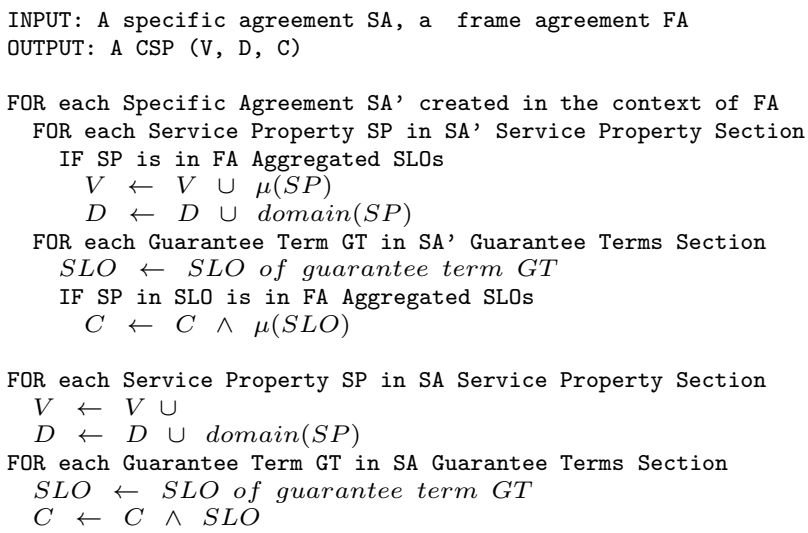

Fig. 2. Algorithm for mapping specific agreements into CSPs

on properties and attributes so it can be described as CSP constraints in a straight way. Second, similar mappings have been successfully used to automate conformance tasks between agreement offer and templates [10]. Finally, there is a plethora of off-the-shelf CSP solvers that can be used to automate this checking.

The mapping step of the procedure involves two different mappings to CSP. One for the specific agreement whose conformance is being checked and the previous specific agreements that have been created in the context of the same frame agreement and another one for the frame agreement itself.

The mapping for specific agreements is depicted in Figure 2, The variables of the CSP are the service properties specified in the agreements and their domains are the domains of the service properties. Regarding the constraints, they are the content of the SLOs of the specific agreements (in the case of previous specific agreements, only of those SLOs that include service properties used in aggregated SLOs in the frame agreement). Note that variables are processed by function $\mu(X)$, which renames service properties according to the agreement it belongs (i.e.: $\mu$ (transittime $<=30)$ in specific agreement 1 returns 'transittime ${ }_{1}<=$ $\left.30^{\prime}\right)$ to avoid collisions of names between the different specific agreements.

The algorithm depicted in Figure 3 applies the same mapping, but now to the elements of the frame agreement. However, in this case, if the SLO uses the aggregation operation, then it is previously processed by a function $\alpha(S L O)$, which unfolds aggregation operations in SLOs according to the specific agreements related to the service property used in the SLO (i.e.: $\alpha(S U M$ containers $<1000)$ returns container $_{1}+$ container $\left.\left._{2}+\ldots<1000\right)\right)$.

The CSPs obtained applying both mappings to example specific agreements and frame agreement is displayed in Table 1. Finally, with these CSP mappings, conformance between specific and frame agreement can be defined as follows:

Definition 1. Let $S A$ be a specific agreement, $F A$ a frame agreement, $\left(V_{s}, D_{s}, C_{s}\right)$ the CSP obtained after mapping $S A$ and previous specific agreements created in the

${ }^{4}$ http://www.emn.fr/z-info/choco-solver/ 


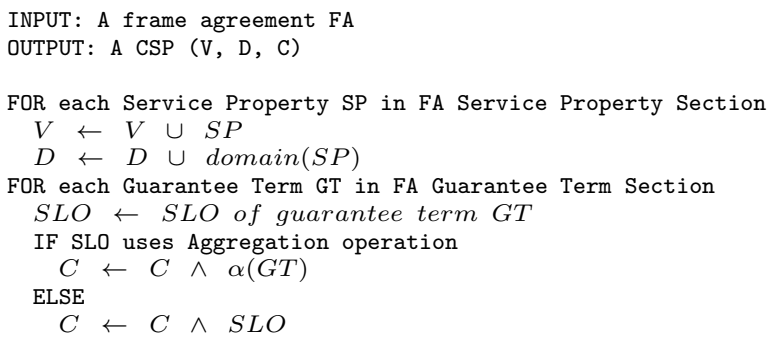

Fig. 3. Algorithm for mapping frame agreements into CSPs

Table 1. Mapping for Example

\begin{tabular}{|l|l|}
\hline Agreement & CSP Mapping \\
\hline Specific Agreements & $V \leftarrow$ A1Containers, Containers, TransitTime \\
(Current and A1) & $D \leftarrow[0,1000],[0,1000],[0,365]$ \\
& $C \leftarrow$ A1Containers $=500 \wedge$ Containers $=600 \wedge$ TransitTime $=23$ \\
\hline Frame Agreement & $V \leftarrow$ Containers, TransitTime, \\
& $D \leftarrow[0,1000],[0,365]$ \\
& $C \leftarrow$ TransitTime $<30 \wedge$ A1Containers + Containers $<=1000$ \\
\hline
\end{tabular}

context of $F A$, and $\left(V_{f}, D_{f}, C_{f}\right)$ the CSP obtained after mapping $F A$. The current specific agreement conforms with the frame agreement if:

$$
\operatorname{conforms}(S A, F A) \Leftrightarrow \neg \operatorname{sat}\left(V_{s} \cup V_{f}, D_{s} \cup D_{f}, C_{s} \wedge \neg C_{f}\right)
$$

The rationale for this definition is as follows. According to the intuitive definition stated above, a specific agreement conforms to a frame agreement if the set of possible values for the service properties used in the SLOs in the specific agreement is a subset of the possible values for the service properties used in the SLOs in the frame agreement. In terms of CSP this can be expressed as:

$$
\operatorname{conforms}(S A, F A) \Leftrightarrow \forall \vec{x} \in V_{s} \cup V_{f} \cdot \operatorname{satisfies}\left(x, C_{s}\right) \Rightarrow \operatorname{satisfies}\left(x, C_{f}\right)
$$

which can be rewritten as:

$\operatorname{conforms}(S A, F A) \Leftrightarrow \neg \exists \vec{x} \in V_{s} \cup V_{f} \cdot \neg\left(\neg \operatorname{satisfies}\left(x, C_{s}\right) \vee \operatorname{satisfies}\left(x, C_{f}\right)\right)$

Finally, as satisfiability operation holds if exists solution for a boolean formula, we can write:

$$
\operatorname{conforms}(S A, F A) \Leftrightarrow \neg \operatorname{sat}\left(V_{s} \cup V_{f}, D_{s} \cup D_{f}, C_{s} \wedge \neg C_{f}\right)
$$

With this definition, the result of validation for the example in Table 1 is false since $\neg($ A1Containers + Containers $<=1000) \equiv 600+500>1000$ is satisfiable. Therefore, conforms $(S A, F A) \equiv$ false.

These operations have been implemented in the ADA (Agreement Document Analyser) Framework using JAVA language and CHOCO solver. The prototype can be accessed in the project URL: http://www.isa.us.es/tlcc. 


\section{Conclusions}

In this paper, the Transport \& Logistics compliance checking has been automated using computational service model, as WS-Agreement specification, and techniques as CSP transforming and solvers. However, the complex artifacts in scenario, as frame agreements, which define rules for consequent specific agreements, require extending WS-Agreement specification with enhanced models to support frame agreements and specific agreements. As new models are defined, new mappings have been introduced to solve the compliance checking with CSP solvers. This proposal supports conformity checking for frame agreement and specific agreement in Transport \& Logistics but as these artifacts appear naturally in other computational and non-computational scenarios. Therefore the enriched model can be the basis to solve other two-level SLA scenarios.

\section{References}

1. Augenstein, C., Ludwig, A., Franczyk, B.: Integration of service models - preliminary results for consistent logistics service management. In: 2012 Annual SRII Global Conference (SRII), pp. 100-109 (2012)

2. Bartolini, C., Bertolino, A., De Angelis, G., Ciancone, A., Mirandola, R.: Apprehensive qos monitoring of service choreographies. In: Proceedings of the 28th Annual ACM Symposium on Applied Computing, SAC 2013, pp. 1893-1899. ACM, New York (2013)

3. Bing, W., Zhongying, L.: Decision-making in optimizing the contract of third party logistic. In: 6th International Conference on Service Systems and Service Management, ICSSSM 2009, pp. 444-449 (2009)

4. Braga, C., Chalub, F., Sztajnberg, A.: A formal semantics for a quality of service contract language. Electronic Notes in Theoretical Computer Science 203(7), 103$120(2009)$

5. Goel, N., Kumar, N., Shyamasundar, R.K.: SLA monitor: A system for dynamic monitoring of adaptive web services. In: 2011 Ninth IEEE European Conference on Web Services (ECOWS), pp. 109-116 (2011)

6. Leitner, P., Ferner, J., Hummer, W., Dustdar, S.: Data-driven and automated prediction of service level agreement violations in service compositions. Distributed and Parallel Databases 31(3), 447-470 (2013)

7. Hua Mai, Y., Xin Miao, L., Teo, C.P., Qingqing, X.: Geometric approach for logistics outsoursing contracting. In: 2010 8th International Conference on Supply Chain Management and Information Systems (SCMIS), pp. 1-7 (2010)

8. Metzger, A., Franklin, R., Engel, Y.: Predictive monitoring of heterogeneous service-oriented business networks: The transport and logistics case. In: Service Research and Innovation Institute Global Conference (SRII 2012). Conference Publishing Service (CPS). IEEE Computer Society (2012)

9. Müller, C., Resinas, M., Ruiz-Cortés, A.: Automated analysis of conflicts in wsagreement. IEEE Transactions on Services Computing PP(99), 1 (2013)

10. Müller, C., Resinas, M., Ruiz-Cortés, A.: Explaining the non-compliance between templates and agreement offers in WS-agreement. In: Baresi, L., Chi, C.-H., Suzuki, J. (eds.) ICSOC-ServiceWave 2009. LNCS, vol. 5900, pp. 237-252. Springer, Heidelberg (2009) 